\title{
A Non-Binary Trellis Code Modulation-PAM-based Novel Decoding Algorithm
}

\author{
Riyadh A. Al-hilali \\ Iraqi affiliation \\ College of Engineering \\ Al-Mustansiriyah University
}

\author{
Abdulkareem S. Abdallah \\ Iraqi affiliation \\ College of Engineering \\ Basrah University
}

\author{
Raad H. Thaher \\ Iraqi affiliation \\ College of Engineering \\ Al-Mustansiriyah University
}

\begin{abstract}
The proposed system deals with Non-binary error control coding of the TCM scheme for transmissions over the AWGN channel. The idea of Non-binary codes has been extended for symbols defined over rings of integers, which outperform binary codes with only a small increase in decoding complexity. The basic mathematical concepts are necessary for working with Non-binary error-correcting codes are Groups, Rings and Fields.
\end{abstract}

A new non-binary decoding method, Yaletharatalhussein decoding algorithm, is designed and implemented for decoding non-binary convolutional codes which is based on the trellis diagram representing the convolutional encoder.

Yaletharatalhussein decoding algorithm outperforms the Viterbi algorithm and other algorithms in its simplicity, very small computational complexity, and easy to implement with real-time applications. The simulation results show that the performance of the non-binary TCM-based Yaletharatalhussein decoding algorithm outperforms the binary and non-binary decoding methods.

\section{General Terms}

Coded modulation, Digital modulation, Groups, Rings of integer.

\section{Keywords}

TCM codes, Non-binary error correcting codes, Convolutional codes, Trellis code modulation.

\section{INTRODUCTION}

Digital signals are more reliable in a noisy communications environment. They can usually be detected perfectly, as long as the noise levels are below a certain threshold. Digital data can easily be encoded in such a way as to introduce dependency among a large number of symbols, thus enabling a receiver to make a more accurate detection of the symbols. This is called error control coding.

Power and bandwidth are limited resources in modern communications systems, and their efficient exploitation invariably involves an increase in the complexity of a communication system. One very successful method of reducing the power requirements without increase in the requirements on bandwidth was introduced by Gottfried Ungerboeck [1,2], subsequently termed trellis-coded modulation (TCM). Further performance gains can be achieved by using non-binary codes in the coded modulation scheme, but with an increase in the decoding complexity [3]. Non-binary codes are the most commonly used error- correcting codes and can be found in optical and magnetic storage, high-speed modems and wireless communications.

When conventional coding techniques are introduced in a transmission system, the bandwidth of the coded signal after modulation is wider than that of the uncoded signal for the same information rate and the same modulation scheme. In fact, the encoding process requires a bandwidth expansion that is inversely proportional to the code rate, being traded for a coding gain.

The basic principle of CM [4] is that it attaches a parity bit to each uncoded information symbol formed by $m$ information bits according to the specific modulation scheme used, hence doubling the number of constellation points to $2^{m+1}$ compared with that of $2^{m}$ in the original modem constellation. This is achieved by extending the modulation constellation, rather than expanding the required bandwidth, while maintaining the same effective throughput of $m$ bits per symbol, as in the case of no channel coding.

As trellis-coded modulation is an extension of binary coding methods to larger signal constellations, so is turbo-coded modulation the extension of turbo coding principles to include larger signal constellations. There are very few changes necessary to accommodate higher signaling alphabets, and therefore higher spectral efficiencies.

Among the various CM schemes, TCM [5] was originally designed for transmission over Additive White Gaussian Noise (AWGN) channels. TTCM [3] is a more recent joint coding and modulation scheme which has a structure similar to that of the family of binary turbo codes, but employs TCM schemes as component codes. Both TCM and TTCM employ set-partitioning-based constellation mapping [6], while using symbol-based turbo interleavers and channel interleavers.

Another CM scheme, referred to as BICM [7], invokes bitbased channel interleavers in conjunction with grey constellation mapping. Furthermore, iteratively decoded BICM [8] using set partitioning was also proposed. R. A Carrasco et. al., 2009 [9] presents the theory of non-binary error control coding in wireless communications.

\section{NON-BINARY TCM}

Convolutional codes and TCM codes are based on rings of integers modulo-M. Due to the similarities between M-PSK signal sets and the algebraic structure of rings of integers modulo-M, modulo-M ring-TCM codes are the natural linear codes for M-PSK modulation.

\subsection{TCM Based on Rings of Integers}

The general structure of a ring-TCM encoder suitable for MPSK modulation, assuming that $\mathrm{m}$ information bits are 
transmitted per baud, with $\mathrm{M}=2 \mathrm{~m}+1$, is shown (see Figure 1). This ring-TCM encoder works as follows [9]:

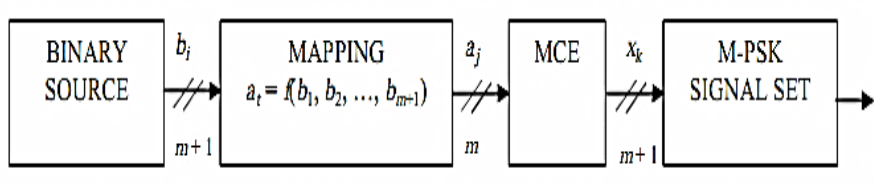

Fig 1. General structure of a ring-TCM encoder suitable for M-PSK modulation

* First, $m+1$ information bits, $b_{\mathrm{i}}$, are mapped into a modulo-M symbol, $\mathrm{a}_{\mathrm{j}}$, according to a mapping function $f$ (for instance, $f$ can be a Gray mapping function).

* Next, $m$ modulo-M $\mathrm{a}_{\mathrm{j}}$ symbols are introduced into a linear multi-level convolutional encoder (MCE), which generates $m+1$ modulo-M coded symbols, $\mathrm{x}_{\mathrm{k}}$.

* Finally, each one of these coded symbols $x_{k}$ is associated with a signal of the M-PSK signal set and is sent to the channel.

As a total of $m+1$ modulo-M coded symbols $\mathrm{x}_{\mathrm{k}}$ are transmitted per single trellis branch, ring-TCM codes can be considered as $2(m+1)$-dimensional TCM codes.

\section{RINGS OF INTEGERS}

If the two binary operations ' + ' and '.' are allowed then a ring can be defined. A ring must have the following conditions; associativity, distributivity, and commutativity under addition. The ring is called a commutative ring if it also has commutativity under multiplication. If the ring has a multiplicative identity 1 then it is called a ring with identity. An example of a ring is the ring of integers $\mathbb{Z}_{q}$ under modulo- $\mathrm{q}$ addition and multiplication, where $\mathrm{q}$ is the cardinality of the ring. For example, $\mathbb{Z}_{4}$ is defined as $\{0,1,2$, $3\}$.

It is easy to see that the elements obey the three definitions of a ring. Also, all the elements commute under multiplication and the multiplicative identity element 1 is present, meaning that $\mathbb{Z}_{4}$ is a commutative ring with identity. Table 1 and Table 2 show the addition and multiplication tables respectively of the ring of integers $\mathbb{Z}_{4}=\{0,1,2,3\}[10]$.

Table 1. Addition table for $\mathbb{Z}_{4}$

\begin{tabular}{|c|c|c|c|c|}
\hline+ & $\mathbf{0}$ & $\mathbf{1}$ & $\mathbf{2}$ & $\mathbf{3}$ \\
\hline $\mathbf{0}$ & 0 & 1 & 2 & 3 \\
\hline $\mathbf{1}$ & 1 & 2 & 3 & 0 \\
\hline $\mathbf{2}$ & 2 & 3 & 0 & 1 \\
\hline $\mathbf{3}$ & 3 & 0 & 1 & 2 \\
\hline
\end{tabular}

Table 2. Multiplication table for $\mathbb{Z}_{4}$

\begin{tabular}{|c|c|c|c|c|}
\hline$\cdot$ & $\mathbf{0}$ & $\mathbf{1}$ & $\mathbf{2}$ & $\mathbf{3}$ \\
\hline $\mathbf{0}$ & 0 & 0 & 0 & 0 \\
\hline $\mathbf{1}$ & 0 & 1 & 2 & 3 \\
\hline $\mathbf{2}$ & 0 & 2 & 0 & 2 \\
\hline $\mathbf{3}$ & 0 & 3 & 2 & 1 \\
\hline
\end{tabular}

The set of all polynomials with coefficients defined in $\mathbb{Z}_{q}$ forms a ring under the addition and multiplication operations.

\section{A 4-STATE RING-TCM CODE DEFINED OVER 4}

A good 4-state ring-TCM code over $\mathbb{Z}_{4}$ is shown (see Figure

2 ) and this would be used in this research work [9].

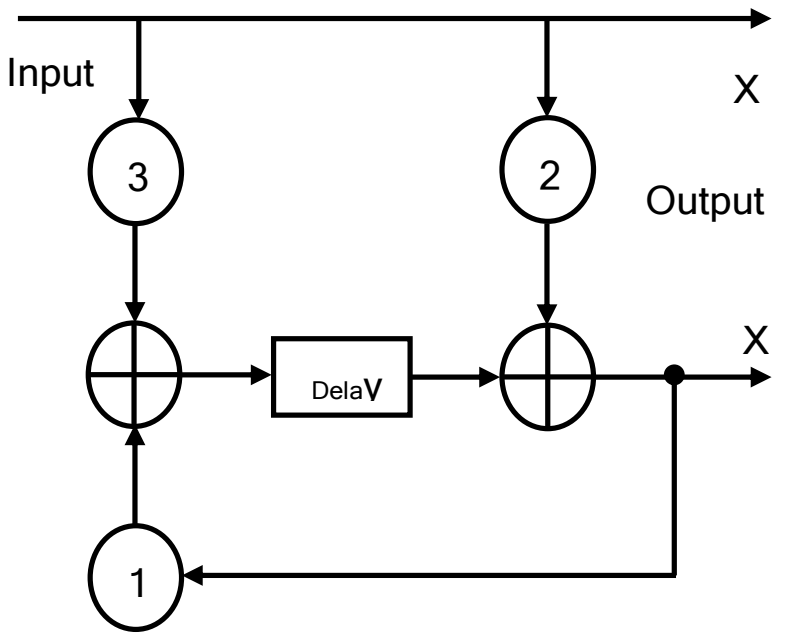

Fig 2. A 4-state ring-TCM Encoder

Where each of the input and output of Fig 2 represents the elements of the ring of integers modulo- $4, \mathbb{Z}_{4}=\{0,1,2,3\}$, and all addition arithmetic operations satisfy the properties of the ring of integers modulo-4 addition. The delay block represents the constraint length (number of memory elements, i.e., in this case there is only one memory element).

\subsection{Yaletharatalhussein Decoding Algorithm}

The Yaletharatalhussein non-binary decoding algorithm is proposed in this paper for decoding non-binary convolutional codes. Convolutional codes differ from block codes in that a block code takes a fixed message length and encodes it, whereas a convolutional code can encode a continuous stream of data, and a hard-decision decoding can easily be realized using the Yaletharatalhussein algorithm. The decoder for the non-binary convolutional code finds the most probable sequence of data bits $\hat{u}$ given the received sequence $y$ :

$$
\hat{u}=\arg \{\underbrace{\max }_{u} p(u \mid y)\}
$$

Where $y$ is the set of code symbols $c$ observed through noise. The above equation can be solved using the Yaletharatalhussein algorithm, explained later.

The principle states that creating a state vector containing binary logic states, which represents the similarities and differences between $y$ symbols associated with each $u$ bits at the current time instant, and then searching for a minimum logic state in this vector to determine the state node number with its order bit for using in the next time instant of searching strategy method, in one hand, and for recovering the transmitted code word in the other hand. In this case, the decoding method is independent on the trellis diagram representing the non-binary convolutional encoder. The $\mathbb{Z}_{4}$ ring-TCM demodulator and decoder-based yaletharatalhussein decoding algorithm can be represented by 
a flowchart as shown (see Figure 3). The flow chart inside the dashed lines represents the yaletharatalhussein decoding algorithm.

\section{SIMULATION RESULTS}

The scatter plots of constellation points, with different values of phase offset, for the $\mathbb{Z}_{4}$-Ring-TCM encoder-modulatorbased PSK schemes are shown (see Fig 4). The values of phase offset angles are; pi/2, pi/4, pi/8, pi/16, pi/32. The performance of the $\mathbb{Z}_{4}$-Ring-TCM scheme-based yaletharatalhussein algorithm communicating over the AWGN channel is shown (see Figure 5).

For the sake of comparison with the proposed non-binary scheme in this thesis, the work [12] was presented the performance of non-binary TCM-QPSK system and can be shown ( see Figure 6), since, TCM code was transparent, and decoded using the soft-decision Viterbi algorithm [13, 14].

The performances of the $\mathbb{Z}_{4}$-Ring-TCM scheme-based Yaletharatalhussein decoding algorithm and the presented 4state ring-TCM code on pedestrian channel [12] can be summarized in Table 3, where the coding gains are defined as the $\left(\mathrm{E}_{\mathrm{b}} / \mathrm{N}_{\mathrm{o}}\right)$ difference, expressed in decibels, at BERs of $10^{-5}$ and $10^{-3}$. The performance of the best scheme in Table 3 is (printed in bold), since the performance comparison shows that the $\mathbb{Z}_{4}$-ring-TCM-PAM scheme-based Yaletharatalhussein algorithm outperforms the scheme [12], since this scheme is provided that the gains in $\left(\mathrm{E}_{\mathrm{b}} / \mathrm{N}_{\mathrm{o}}\right)$ are $(17.61 \mathrm{~dB})$ and $(34.82 \mathrm{~dB})$ at the BERs of $10^{-3}$ and $10^{-5}$ respectively.

Also, the $\mathbb{Z}_{4}$-ring-TCM-PAM scheme-based Yaletharatalhussein algorithm outperforms the 4-state ringTCM-based Viterbi algorithm by the gains $(8.7 \mathrm{~dB}$ and 5.5 $\mathrm{dB})$ at the BERs $\left(10^{-3}\right.$ and $\left.10^{-5}\right)$ respectively.

\section{CONCLUSIONS AND FUTURE WORKS}

A novel non-binary decoding method, is called Yaletharatalhussein decoding algorithm, is proposed for decoding non-binary convolutional and TCM codes, which independent on the trellis diagram representing the nonbinary convolutional encoder, as in Viterbi algorithm.

The Yaletharatalhussein algorithm employed a hard-decision decoding, which needed less computational complexity over the soft-decision MLD of Viterbi algorithm.

In Yaletharatalhussein algorithm, the code words are detected instantaneously through searching in the developed state vectors, while in Viterbi algorithm, the hamming distances between numbers and transition metrics are calculated and a comparison between competitive accumulated metrics is done for every state of the trellis diagram.

In Yaletharatalhussein algorithm, the massages are decoded in blocks reached to 1000,000 symbols as seen in simulation results, while in a typical Viterbi code, messages are decoded in blocks of only about 200 bits or so, whereas in turbo coding the blocks are on the order of $16 \mathrm{~K}$ bits long. Nonbinary TCM codes outperform binary TCM codes with a small increase in decoding complexity.
A future work can be done by applying the Yaletharatalhussein decoding algorithm to higher order rings of integers such as, $\mathbb{Z}_{8}, \mathbb{Z}_{16}, \mathbb{Z}_{32}$, and $\mathbb{Z}_{64}$, and then study the error performance and bandwidth efficiency of the system for different types of modulation schemes and orders; 8, 16, 32, and 64.

\section{REFERENCES}

[1] D. Pham, K. R. Pattipati, P. K. Willett, and J. Luo, 'An improved complex sphere decoder for V-BLAST systems', IEEE Signal Processing Letters, vol. 11, pp. 748-751, September 2004.

[2] F. Adachi and K. Ohno, 'BER performance of QDPSK with postdetection diversity reception in mobile radio channels', IEEE Transactions on Vehicular Technology, vol. 40, pp. 237-249, February 1991.

[3] Bahl, L. R., Cocke, J., Jelinek, F., Raviv, J. Optimal decoding of linear codes for minimizing symbol error rate. IEEE Trans. Inform. Theory, vol. 20, pp.284-287, 1974.

[4] 'Using MIMO-OFDM Technology To Boost Wireless LAN Performance Today', White Paper, Data comm Research Company, St Louis, USA, June 2005.

[5] H. Sampath, S. Talwar, J. Tellado, V. Erceg, and A. J. Paulraj, 'A fourth-generation MIMO-OFDM broadband wireless system: design, performance, and field trial results', IEEE Communications Magazine, vol. 40, pp. 143-149, September 2002.

[6] WiMAX Forum, 'WiMAX Forum WiMAX Technology Forecast (2007-2012)', June 2008. http://www.wimaxforum.org/technology/downloads/.

[7] Haohong W., L. P. Kondi, Ajay L., and Song Ci, 4G Wireless Vedio Communications. United Kingdom, John Wiley \& Sons Ltd, first published 2009.

[8] S. Hara and R. Prasad, Multicarrier Techniques for 4G Mobile Communications. Boston, Artech House, 2003.

[9] Carrasco, R., Lopez, F. and Farrell, P. Ring-TCM for M-PSK modulation: AWGN channels and DSP implementation. Communications, IEE Proceedings, 143, 273-80, 1996.

[10] E. H. Connel, Elements of Abstract and Linear Algebra. Coral Gables, Florida 33124 USA, 2004.

[11] Naguib, A., Tarokh, V., Seshadri, N. and Calderbank, A. A space-time coding modem for high-data-rate wireless communications. Selected Areas in Communications, IEEE Journal, 16, 1459-78, 1998.

[12] Viterbi, A.J. Error bounds for convolutional codes and asymptotically optimum decoding algorithm. IEEE Transactions on Information Theory, 13, 260-9, 1967.

[13] Forney, G.D. The Viterbi algorithm. Proceedings of IEEE, vol. 61, 268-78, 1973. 


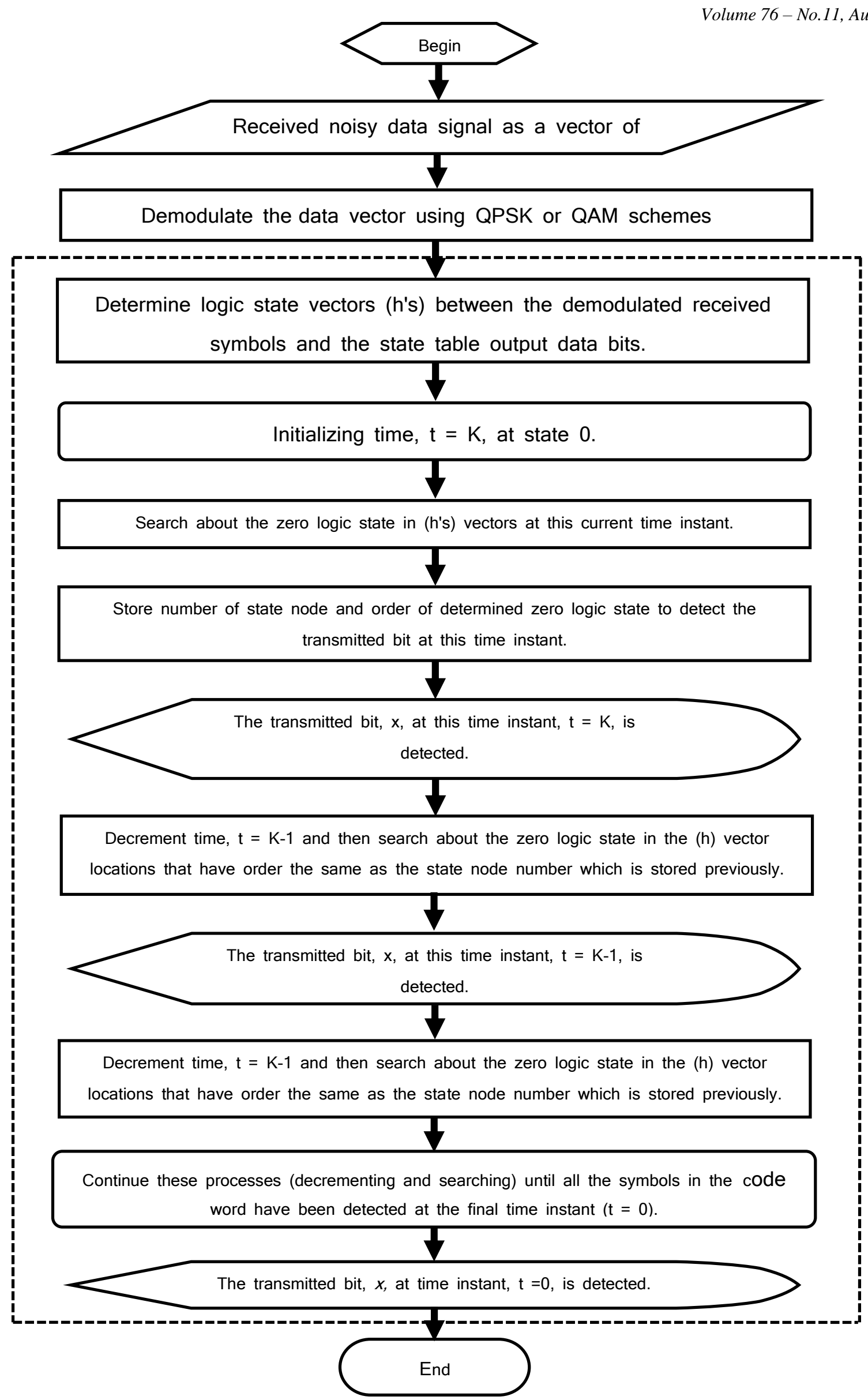

Fig 3. Flow chart of the $\mathbb{Z}_{4}$-Ring-TCM demodulator- decoder-based yaletharatalhussein decoding 

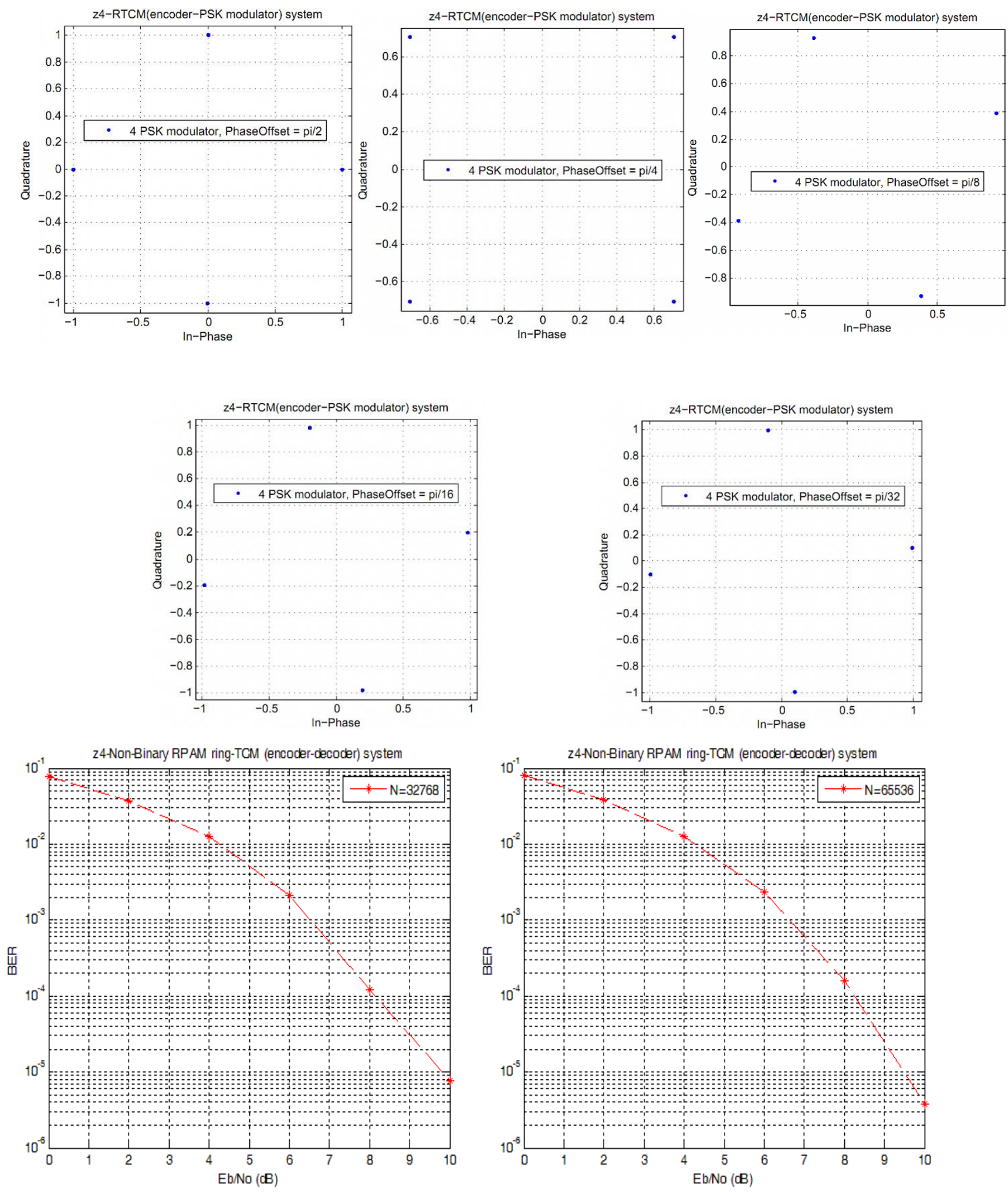

Fig 5. The (BER) versus $\left(E_{b} / N_{0}\right)$ performance of the $\mathbb{Z}_{4}$-Ring-TCM-PAM scheme-based Yaletharatalhussein algorithm. 

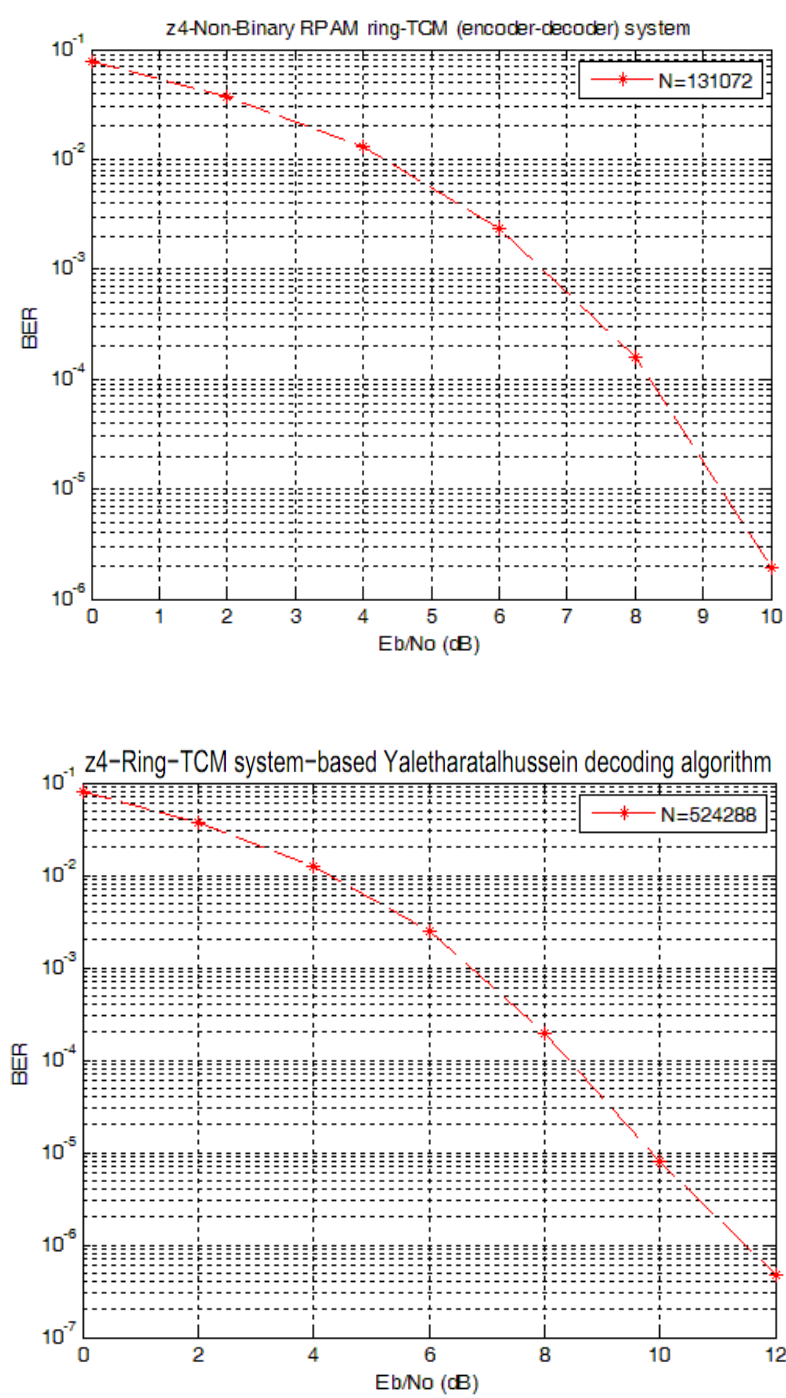
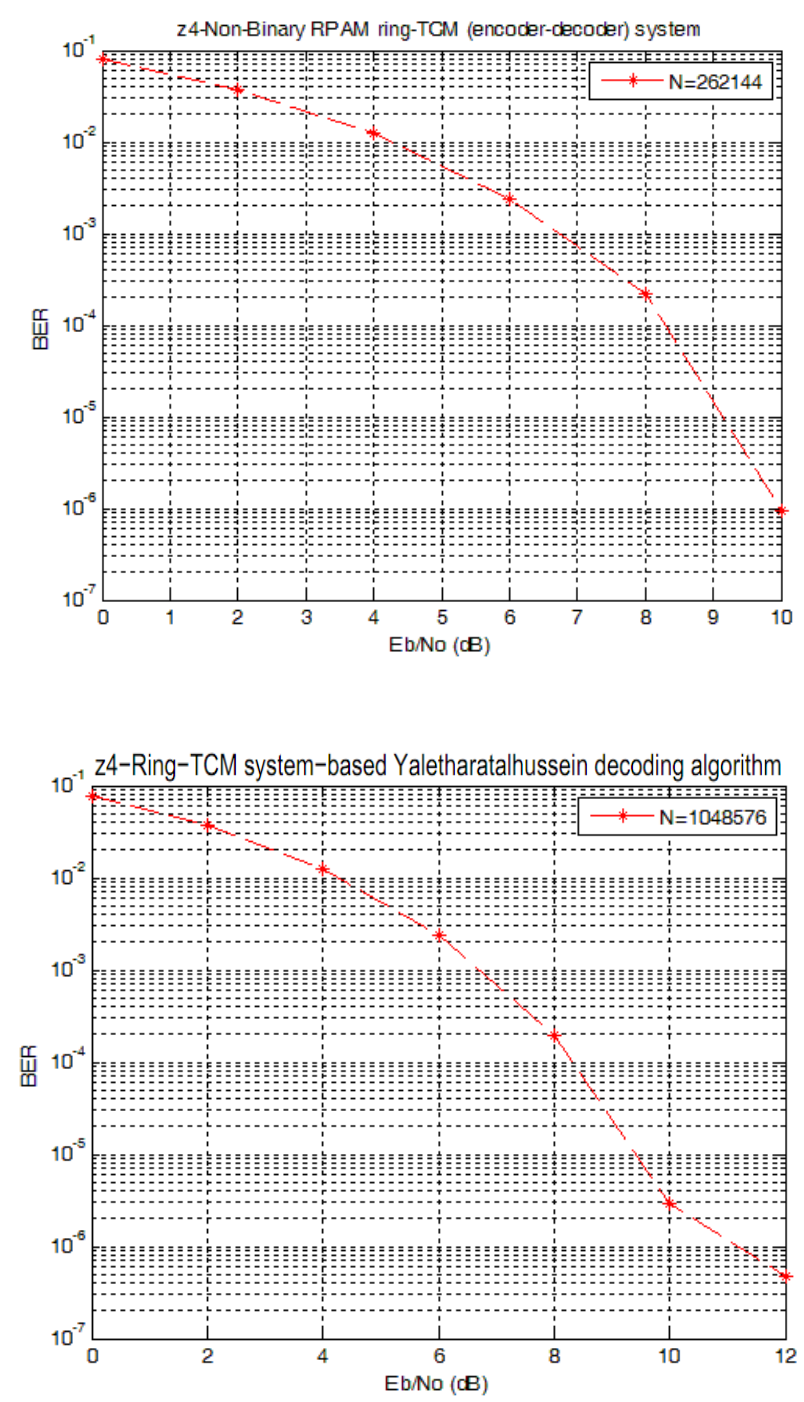

Fig 5.

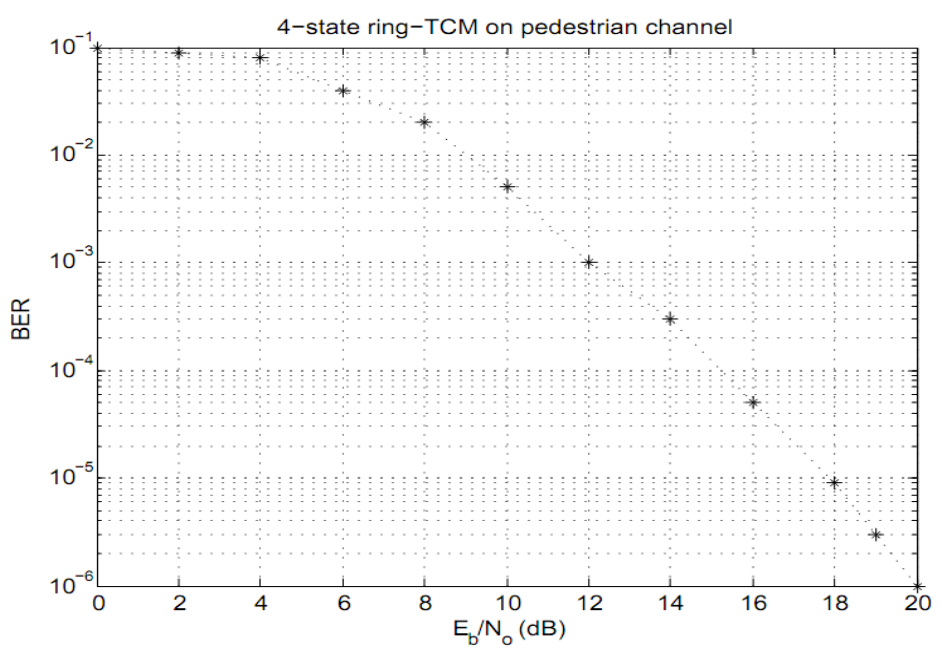

Fig 6. The (BER) versus $\left(E_{b} / N_{0}\right)$ performance of a 4-state ring-TCM code on pedestrian channel [12]

Table 3. Performances of the $\mathbb{Z}_{4}$-Ring-TCM scheme-based Yaletharatalhussein decoding algorithm and the presented 4- 
state ring-TCM code on pedestrian channel [12]

\begin{tabular}{|c|c|c|c|c|c|c|}
\hline & & & \multicolumn{4}{|c|}{ BER } \\
\hline & & & \multicolumn{2}{|c|}{$\mathbf{E}_{\mathbf{b}} / \mathbf{N}_{\mathbf{o}}(\mathbf{d B})$} & \multicolumn{2}{|c|}{ Gain (dB) } \\
\hline $\begin{array}{c}\text { CM } \\
\text { scheme }\end{array}$ & $\begin{array}{c}\text { CM } \\
\text { Code rate }\end{array}$ & $10^{-3}$ & $10^{-5}$ & $10^{-3}$ & $10^{-5}$ & Modem \\
\hline & & 24.11 & 44.12 & 0.00 & 0.00 & BPSK \\
\hline $\begin{array}{l}\mathbb{Z}_{4} \text {-RTCM-based } \\
\text { Yaletharatalhussein algorithm }\end{array}$ & $1 / 2$ & 6.5 & 9.3 & 17.61 & 34.82 & 4-PAM \\
\hline $\begin{array}{l}\text { 4-state RTCM-based Viterbi } \\
\text { algorithm }\end{array}$ & $1 / 2$ & 12 & 18 & 12.11 & 26.12 & QPSK \\
\hline
\end{tabular}

